\title{
Remote Monitoring System for Intelligent Irrigation in Hybrid Wireless Sensor networks
}

\author{
Xiaoqing Yu, Wengting Han* and Zenglin Zhang \\ 1. Northwest A \& F University, Shaanxi, Yangling, 712100, China \\ 2. Research Institute of Water-saving Agriculture of Arid Regions of China, Shaanxi, \\ Yangling, 712100, China \\ yuxiaoqing115@gmail.com;wentinghan200@126.com;zhangzenglin115@gmail.com
}

\begin{abstract}
Aiming at make effective use of variables control, wireless sensor and monitoring, embedded and wireless communications technology in industrial fields to make intelligent precision irrigation in crops the article designed a closed-loop remote monitoring system of distributed precision irrigation by using Citect configuration software, hybrid sensor networks including wireless sensor networks (WSN) and wireless underground sensor networks (WUSN), ARM9 microprocessor and General Packet Radio Service (GPRS) module. The system consists of monitor computer, irrigation monitor controller, wireless sensor networks, GPRS wireless communication module and valves, in which the wireless sensor networks adopt hybrid topology above and below ground, the controllers utilizes embedded technology to control irrigation water yield that applied to each specific unit according to soil water content. The Citect configuration is used to manage data and HMI by the remote monitoring center, which can conduct a more high-level monitoring through web publishing. The results showed that the data sampling interval is set for 30 min, 4 groups WSN nodes were chosen, which the system could get soil temperature, humidity and soil water content in $5 \mathrm{~cm}$ and $35 \mathrm{~cm}$ depth, intelligent irrigation can save water about $25 \%$ compare with normal irrigation. This system realizes online automatic monitoring of crop precise irrigation, a hybrid wireless sensor network model for both aboveground and underground is creatively established, which is also the foundation for further study.
\end{abstract}

Keywords: Wireless sensor networks, ARM9, IRRigation, GPRS, Monitoring system

\section{Introduction}

Facing the gradual lack of water resources and relative backward of irrigation modes in China, research and extension of water saving and semi-precise irrigation technology is increasingly becoming important, and the measurement and control method is the core of water-saving and semi-precise irrigation technology [1]. At present, comparative mature semi-precise irrigation and measurement and control technology is researched and applied in the international conduct real-time monitoring, transmission and feedback of soil parameters by using wired and wireless sensor network [2], moreover, it completes real-time control of terminal water to drip irrigation or sprinkler irrigation and realizes water-saving and semiprecise irrigation.

The United States, Japan, Israel and other countries are international leaders in irrigation measurement and control technology, at the same time, their technical research and consistency of application are obvious [3-5], namely layout method and form of sensor network hardware in the cultivated land are basic same. Normally, in order to undertake semi- 
precise irrigation, they will decorate several wired or wireless soil moisture sensor at a certain depth layer of crops cultivated land surface based on the species, to form wired or wireless sensor network and according to the related transfer protocol, computer software and hardware and irrigation valves, the loop intelligent irrigation monitoring system is made up [6-8]. Although such irrigation monitoring system is not only the research frontiers and hot in the field of international water-saving irrigation, but also the most used system for popularizing and applying in some agriculture developed countries, and is still in research and preliminary promotion stage in our country, the arrangement of the sensors in the cultivated land level is one-dimensional plane form in this system, it can only get a root point water requirement of crops along the soil depth. According to plant physiology characteristic information, each part of soil information of the root affects crop growth. Combining crop physiology and soil information, the collection of more soil water information along crops root becomes the forefront and direction of research. However, there is still not the concrete research of this aspect. Wireless sensor topological organization of a three-dimensional WSN (wireless sensor network) and WUSN (Wireless underground sensor network) [9, 10] is decorated in the soil of crop growth is the focal point of studies and design in this paper.

The research area is the Irrigation test bed of northwest A \& F university in yangling, shaanxi province, which is located at southeast of yangling shaanxi, north latitude $34^{\circ} 18^{\prime}$, east longitude $108^{\circ} 5^{\prime}$, the average altitude of elevation is $480 \mathrm{~m}$. The Test area is 100 square meters, drip, the vertical distance of wireless sensor node along soil depth from $200 \mathrm{~mm}$ shall prevail. Finally, upper nodes are decorated based on ZigBee agreement, while the lower nodes complete communications by using low frequency nRF905 module.

\section{Composition of Remote Monitoring System}

Normally, farmland area is large, the terrain is complex and the environment of farmland operating is bad, so the general irrigation monitoring equipment cannot conduct reliable and normal precise assignments, but WSN has become effective methods of crop water demand information collection and transmission for its characteristics of high density with its node, large range and strong dynamic [11-13]. Although the communication ability of WSN single node is limited, at the same time, GPRS wireless transmission network have Internet delay [14], but the combination of WSN and GPRS is an economic and reasonable method to realize information remote transmission for agricultural irrigation system of data throughput lesser and real-time relative low. The structure of system is shown in Figure 1, it is composed of upper monitor computer, embedded microcontroller controller, GPRS module, wireless sink module and hybrid wireless sensor networks. Based on different depth of WSN monitoring network in soil, this design uses 30 tiny wireless sensor network nodes as terminals of farmland environment perception, node completed collection and transmission of farmland environmental information through agreement, then it will converge data at EZ510 gateway, finally, the data is sent to the ARM controller or the upper monitor machine or end users through a global mobile communications network. 


\section{Control center}

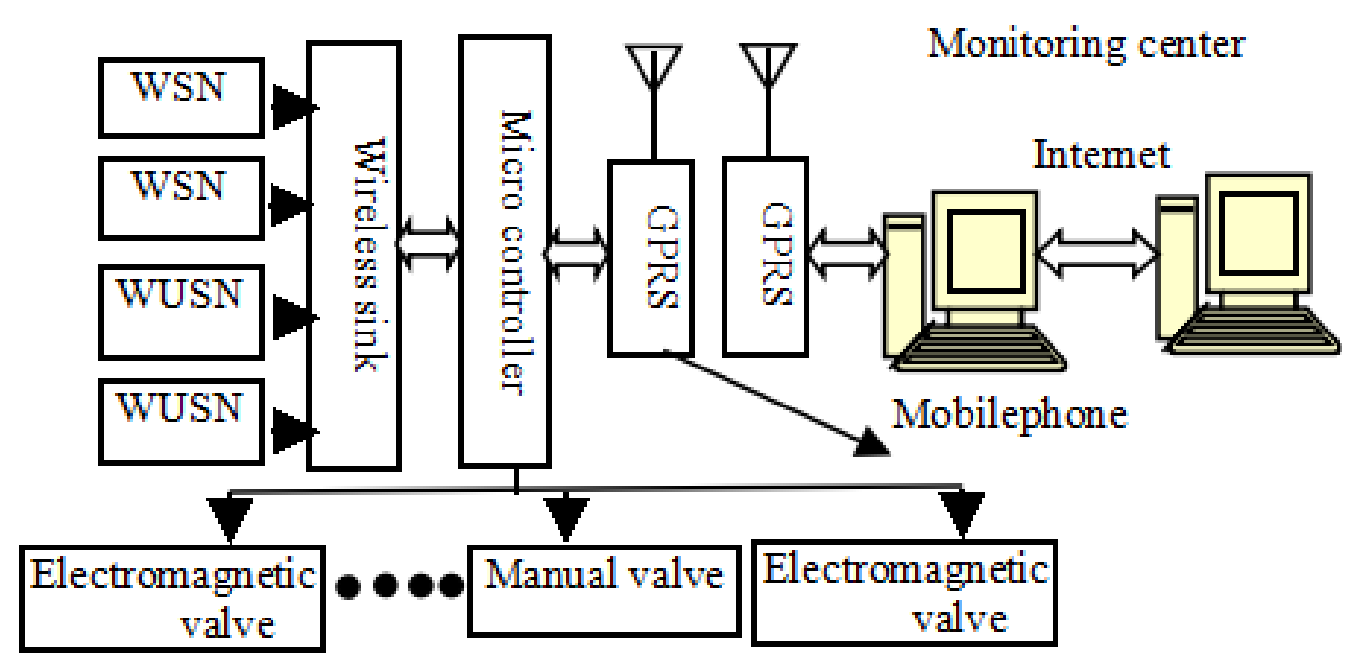

Figure 1. Structure of Remote SCADA System for Intelligent Irrigation

\section{System Principle and Hardware Structure}

\subsection{Working Principle of the System}

Microcontroller system collects temperature, humidity, moisture content and many kinds of soil information through multiple sensors. Then it sends the soil information to the control center hosting through wireless transmission agreement and transmit to upper machine through GPRS. When PC get a certain soil information, it compares and processes automatically these soil information through algorithm and program and gets irrigation instructions, here, these irrigation instruction are fed back to the control center to execute control for valve processor. Among, WSN uses wireless transceiver module CC2420 based on ZigBee agreement [15] to realize soil information collection, WUSN uses nRF905 wireless chip to complete information collection and transmission.

The System realize the precise automatic irrigation control of farmland utilizing sensor network, the WSN signal information of output is transmitted to the control center through wireless full-duplex digital transceiver module to determine whether startup electromagnetic valve for water supply, while the information of whether or not the water is supplied is transferred through Internet by GPRS communication to remote control center to realize the remote monitoring, finally, these information are processed through some model of the computer and then the water supply plan is made.

\subsection{Hardware Structure of the System}

The system consists of two sub-systems. One is control center, which is composed of control host and wireless sensor network nodes. The other is the remote control system, which is formed of GPRS communication module, Internet network transmission and the monitoring center host. Host and wireless sensor network nodes constitute a star topology wireless network, while remote control system implements Internet access by GPRS module to remote control center. The hardware structure diagram of system is shown in Figure 2. 


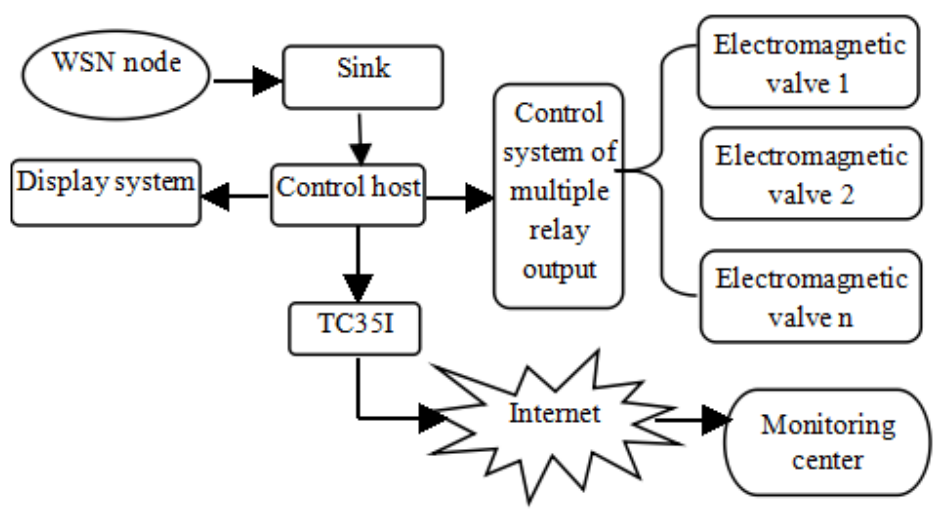

Figure 2. Architecture of System Hardware

\subsection{Design of Wireless Sensor Network Node}

The design of the wireless sensor node uses the modularizing design method, the architecture of ground WSN is shown in Figure 3, underground WSN uses nRF905 wireless chip instead of CC2420 RF chip, the system structure of the entire nodes composed of sensor module, processor module, wireless communication module and energy supply module.

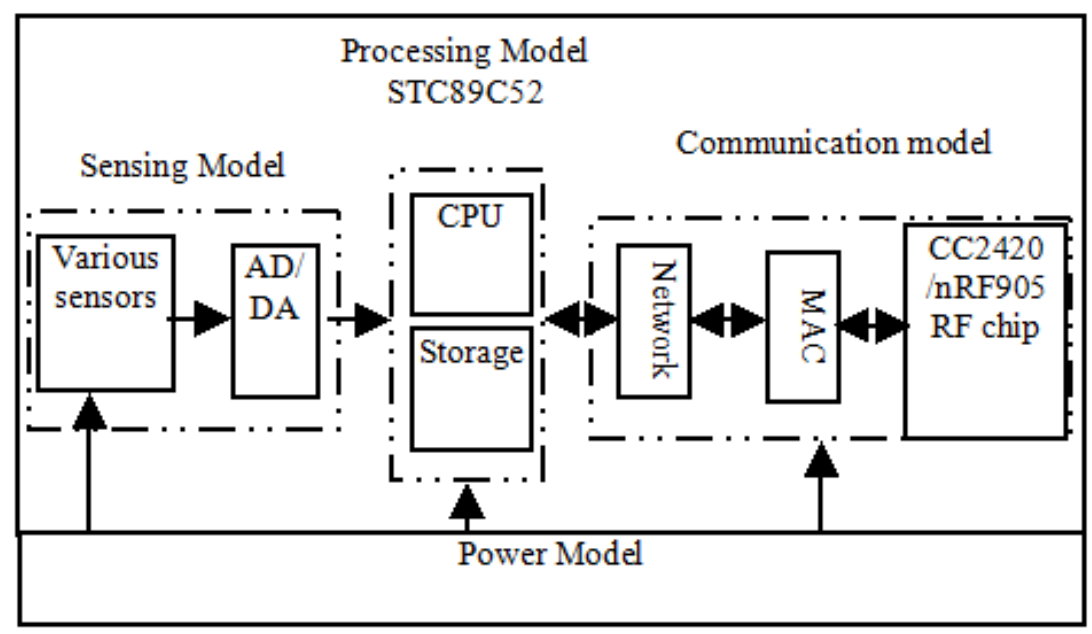

Figure 3. Architecture of Wireless Sensor Network Node

Network topology structure is the basis of the realization of different agreement, a good design of network topology structure should consider the specific application, and also make the implementation of the network protocol simpler, reliable and effective. This design uses mixed wireless sensor network structure of the ground and underground, namely traditional WSN is adopt in underground within $20 \mathrm{~cm}$ deep, while WUSN is adopt in more than $20 \mathrm{~cm}$ deep. Sink is only laid on the ground in WUSN, all nodes in underground will data transmit eventually to the ground sink node, which can make the whole network be better concealment. WUSN node is set depends on the specific application. It can be displayed in the same depth, also can be displayed in different depth and even can be set as layer. The sink node uses fixed or movable, which keep in the range of communication, the topological structure as shown in Figure 4. 


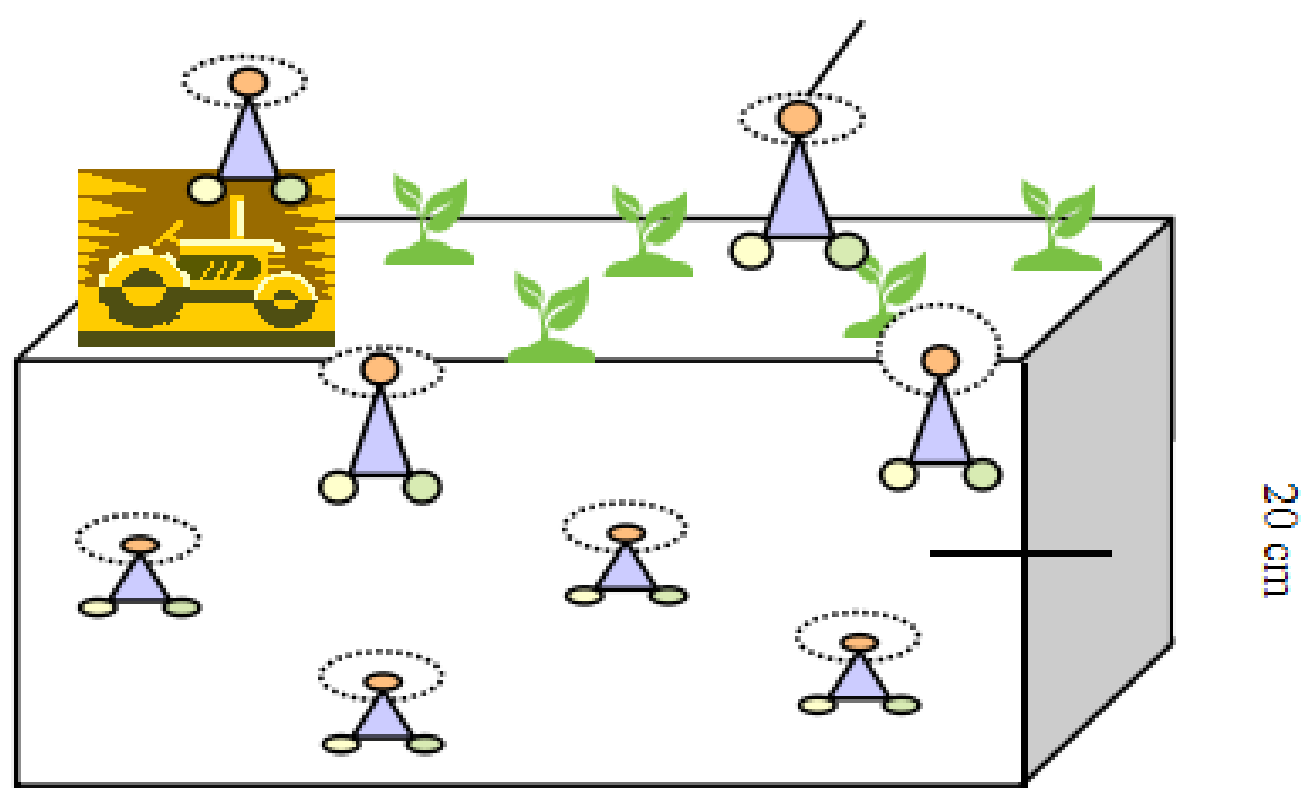

Figure 4. Topology Structure of Wireless Sensor Network

3.3.1. Design of Sensor Module: Sensor module is used to realize data collection of each node. Processor module retains interface to carry various sensors. This design take digital temperature sensor DS18B20 as example, DS18B20 is produced by Dallas company of the United States, which is one of the single line digital temperature sensor, it has many advantages such as miniaturization, low power consumption, high performance, strong antiinterference ability and is easy to match microprocessor [16], the sensor can make temperature directly convert serial digital signal which is processed by processor. Table 1 summarizes the features of the sensors for system using, DS18B20 temperature gathering flow chart is shown in Figure 5.

Table 1. Features of the Sensors

\begin{tabular}{ccc}
\hline Sensor category & Output voltage/V & Measuring range \\
\hline Soil moisture & $0-1.875$ & $0-100 \%\left(\mathrm{~m}^{3} / \mathrm{m}^{3}\right)$ \\
Soil temperature & $0-5$ & $-55-125^{\circ} \mathrm{C}$ \\
\hline
\end{tabular}

3.3.2. Design of Processor Module: STC89C52 microcontroller produced by STC company is taken as the main control chip, this microcontroller has 4 groups I/O of 8 bits (DIP - 40 encapsulation), 3 timing/counter of 16 bits, 8 interrupt sources, 8KB Flash program memory, 512 Byte RAM data memory within slice and a full-duplex serial communication interface. STC microcontroller is high cost-effective, at the same time, the functions are varied, the antiinterference ability is strong, the serial programming is very convenient, moreover, the secrecy is very strong. Schematic diagram is shown in Figure 6. 


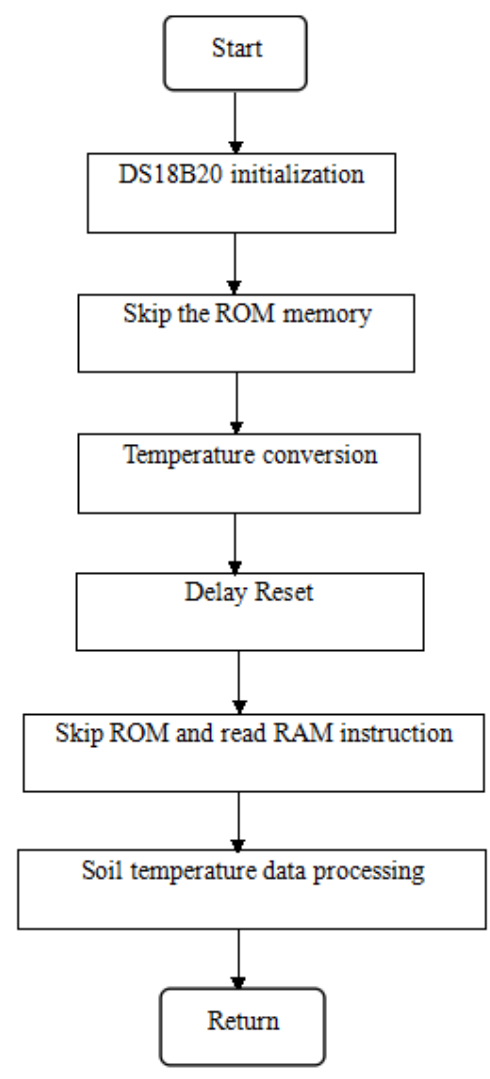

Figure 5. Temperature Gathering Flow of DS18B20

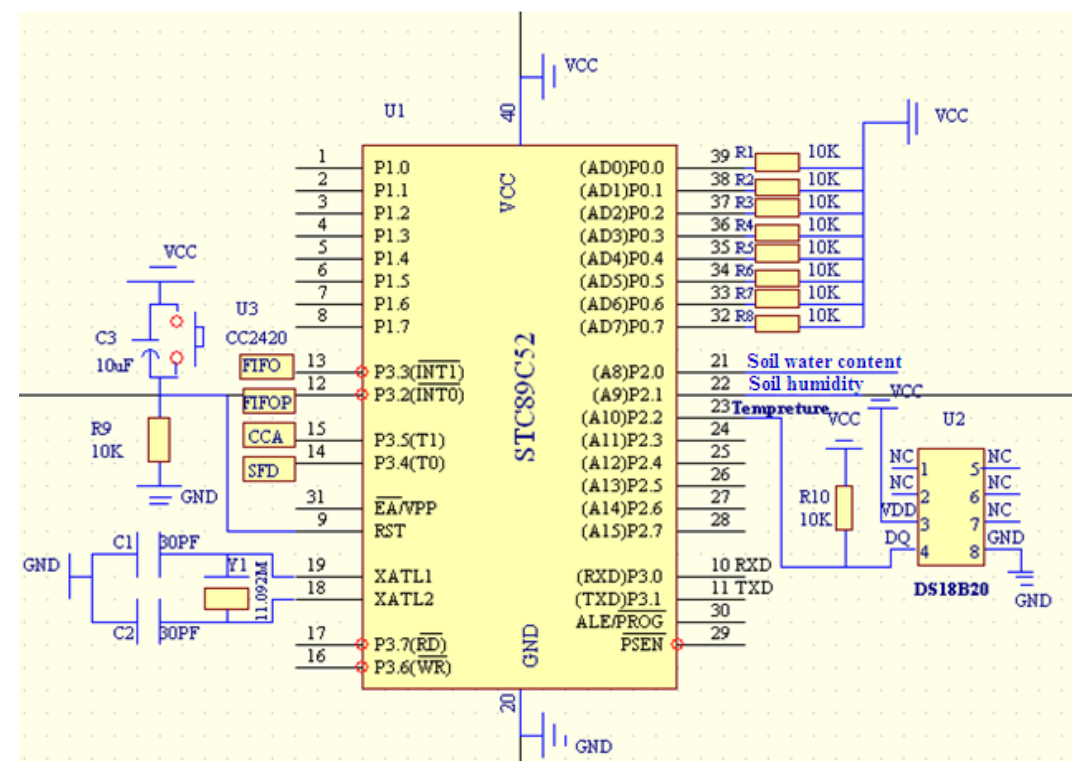

Figure 6. Diagram of Processor Block

3.3.3. Design of Wireless Communication Module: Wireless communication module implements the communication between nodes. RF chip adopts the wireless transceiver chip CC2420 of Chipcon company in WSN which compatible 2.4 GHz IEEE802.15.4 agreement, 
this chip is low voltages when working, low energy [17-18] consumption and little volume features, besides it has the characteristics of output strength and receiving frequency programmable, its external uses the interface similar to SPI, which can connect directly with micro controller, it is very suitable for applications in low energy and small volume, Figure 7. shows CC2420 module.

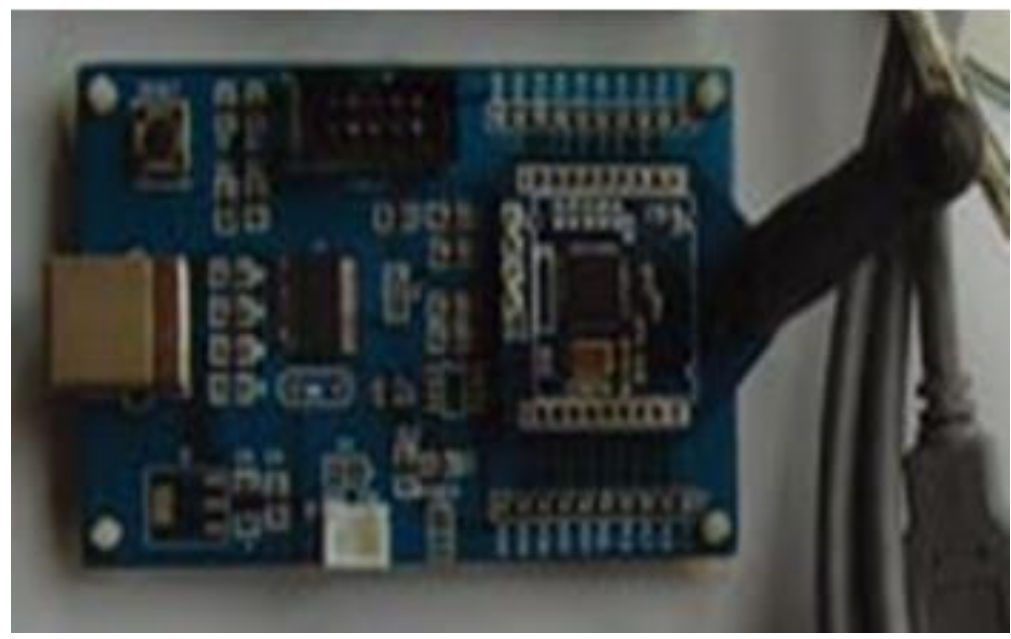

Figure 7. Model of CC2420

In WUSN, the wireless communication chip nRF905 introduced by Nordic companies is used. The chip has two working modes and two energy-saving modes, they are power lost mode, standby mode, ShockBurst TM receiving mode and ShockBurst TM send mode. The chip can work freely in 3 ISM channel which is 433/868/915 MHz. The chip communicates with micro controller through SPI interface, its configuration is very convenient and the power consumption is also very low. Communication circuit with the processor is shown in Figure 8.

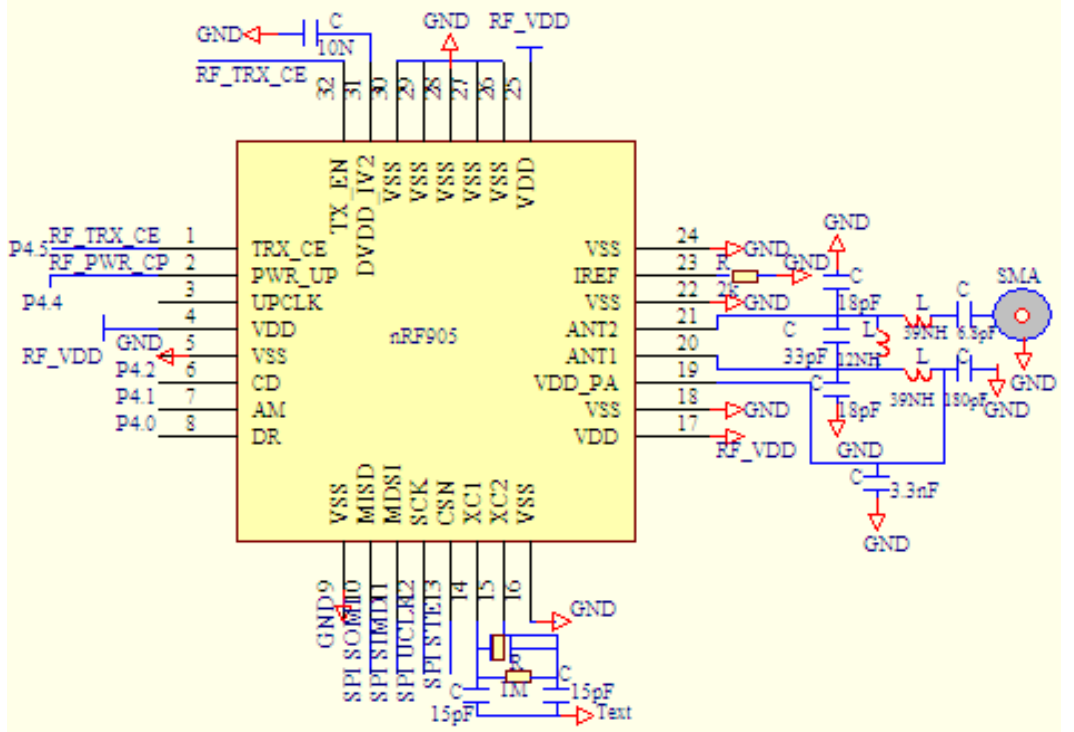

Figure 8. Control Diagram of nRF905 Block 


\subsection{Control Center Module}

The System selects S3C2410 embedded microcontroller of 16/32-bit RISC based on ARM920T kernel. ARM920T nuclear has three parts, which are ARM9TDMI, memory management unit (MMU) and cache separately $[19,20]$. The Control terminal part of system adopts hardware design method of core board and the backplane, the core board integrates S3C2410 microprocessor of Samsung (ARM920T kernel of 32 bits), 16M FALSH, 64M SDRAM and JTAG2ICE debugging interface [21], etc. Resources of core board have 2 UART, a USB port, cable and LCD screen interface. The backplane includes power circuit, all sorts of interface circuit, touch screen, small keyboard, USB storage circuit, etc. Hardware structure of system is shown in Figure 9.

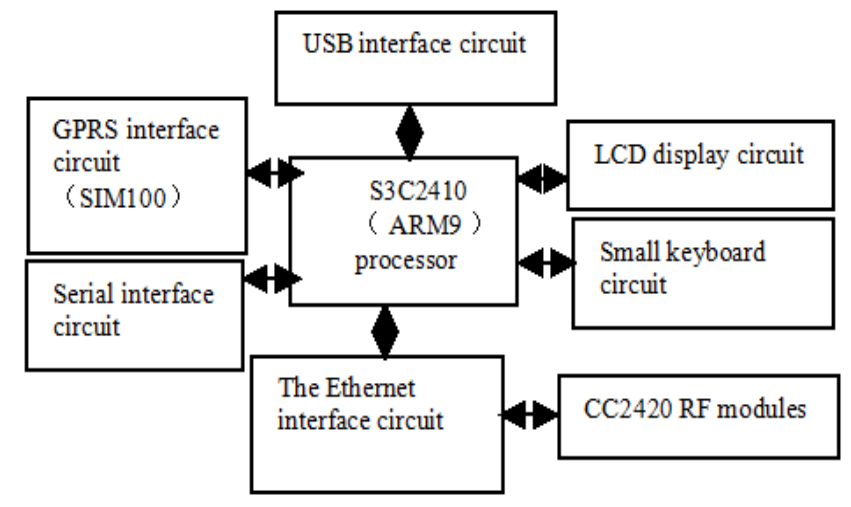

Figure 9. Hardware Structure of Controller Gateway

Sink node uses EZ510 gateway module which connect with microprocessor through a serial port and use external $5 \mathrm{~V}$ power for current supply. Sink node completes program download and all functions include upload of wireless sensor network data. GPRS module completes communication with mobile phone and PC users through GPRS networks. GPRS module uses the TC35I module, which launched by Siemens is a new generation wireless communication GPRS module. It brings RS232 communication interface, which can communicates conveniently with PC and single-chip microcomputer. TC35I can realize fast, safely and reliably data and voice transmission of system solutions, short message service and fax.

The Communication between Micro controller and GPRS module is realized mainly through serial communication and AT instructions [22]. RS232 level signal is used in the process of TC35I serial communication, but signal used is TTL in the process of microcontroller communication, so a data transfer is conducted through MAX232 in the process of communication. All of TC35I module communications adopt AT + XXX format to complete, microcontroller send AT and corresponding control instruction directly through serial communication in the process of communication, which can achieve the communication with TC35I.

\section{Development of System Support Software}

Keil $\mathrm{C}$ programming is used in system microcontroller program, which realizes mainly short-range wireless transceiver function based on ZigBee technology, and communication between wireless module CC2420, nRF905 and GPRS module, GPRS module and GPRS 
module between and open and close of solenoid valves. Finally, it makes the microcontroller complete function management for wireless sensor networks and GPRS network.

The supervisory computer uses industrial control configuration software Citect based on Windows platform developed by Australian CiT company. The Citect system has a good development environment, strong communication protocol support, real-time network data and efficient complete Cicode monitoring language and function sets, which supports operation of real-time database. It utilizes fully the Windows system functions to enrich greatly show scenes and programming environment of the control system and realizes the operating of multitasking [23].

Human-computer interface displays control system plan and process flow diagram in this design by way of graphic pictures. HMI displays dynamic real-time parameters such as soil temperature, humidity and water content signals of each wireless sensor network node in the ground and underground, it also displays real-timely valve running state, accident alarm information list and electrical operation status, these pictures can switch over dynamically and make real-time trend curve and historical data trend curve to process parameter values. Citect configuration software realizes web remote control through ActiveX and CTAPI function, as shown in Figure 10.

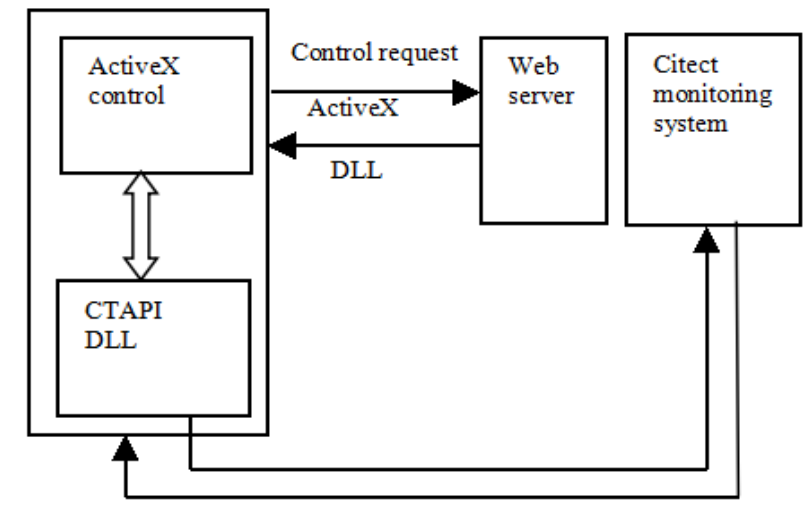

Figure 10. Web Remote Control of Citect System

\section{System Debug}

Data sampling interval is set for $30 \mathrm{~min}$ in test for 2 groups WSN and WUSN nodes. The collecting data can be transmitted to remote PC server through gateway node. Management software installed at the server can realize switching between automatic and manual, storage and display of data, statistical analysis, inquiry, alarm and printing, etc. Trials were measured in the depth of $5 \mathrm{~cm}$ and $35 \mathrm{~cm}$ for soil temperature, humidity and soil water content. Among them, node 1 are $25.700 .80,0.90$, node 2 are $24.00,0.600 .75$, node 3 are 21.60, 0.70, 0.85, node 4 are $20.80,0.55,0.70$, respectively. In addition, node 1 and 3 adopt manual control mode for collecting of soil information in the depth of $5 \mathrm{~cm}$ and $35 \mathrm{~cm}$ respectively, the electromagnetic valve indicator show green, no alarming, so irrigation is not need. Furthermore, node 2 and 4 adopt automatic control mode for collecting of soil information in the depth of $5 \mathrm{~cm}$ and $35 \mathrm{~cm}$ respectively, the electromagnetic valve indicator light show red, alarming is triggered, then the system would open immediately valve for irrigation. Test shows, Comparison with manual irrigation, intelligent irrigation can save water about $25 \%$ and achieve the purpose of water-saving irrigation. Remote monitoring page of the system client and real-time display of monitoring data are shown in Figure 11, the system works well. 


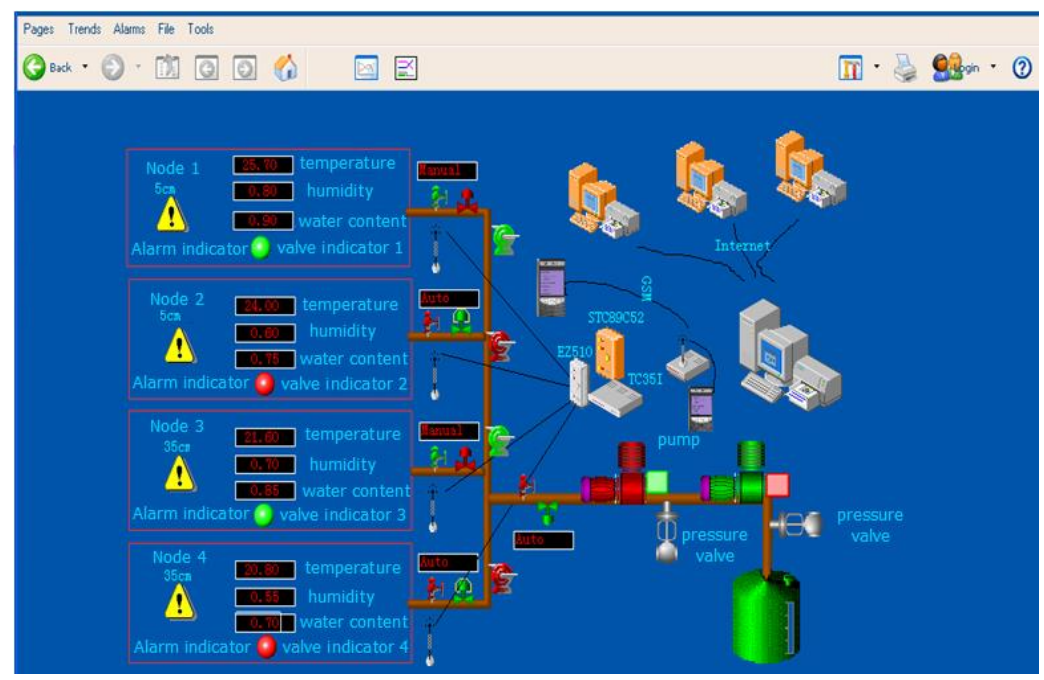

Figure 11. Configuration Chart for System Monitor

\section{Conclusions}

This research is a first step towards modeling based on ground and underground in agriculture. Specifically, it proposed a hybrid wireless sensor networks. According to the application requirement of farmland environmental information, wireless underground sensor network technology is researched. The Underground sensor node and sink node are developed combined with embedded processors, which realized real-time dynamic collection, transmission, store and display for the farmland soil information parameter.

The paper addresses a fundamental design issue in hybrid sensor networks. It has designed a closed-loop remote monitoring system of distributed precision irrigation by using Citect configuration software, hybrid sensor networks including WSN and WUSN, ARM9 microprocessor and GPRS module.

\section{Acknowledgements}

The authors wish to thank the National Engineering Research Center for Water-Saving Irrigation, which partially supported this research through the" China Postdoctoral Science Foundation funded project" and the Program for New Century Excellent Talents in University (NECT-12-0473) from the Ministry of Education. The authors are also grateful to the anonymous reviewers for their valuable feedback.

\section{References}

[1] X. J. Qiao, X. Zhang and C. Wang, "Application of the wireless sensor networks in agriculture", Transactions of the CSAE, (2005), pp. 232-234.

[2] J. Burrrel, T. Brooke and R. Beckwith, "Vineyard computing: sensor networks in agriculture production. Persvasive Computing, vol. 3, no. 1, (2004), pp. 38-45.

[3] T. Fukatsu and M. Hirafuji, "Field Monitoring Using Sensor- Nodes with a Web Server", Journal of Robotics and Mechatronics, vol. 17, no. 2, (2005), pp. 164-172.

[4] G. Sebastia, "Lifetime Issues in Wireless Sensor Networks for Vineyard Monitoring", 2006 IEEE International Conference: Mobile Adhoc and Sensor Systems (MASS). Washington DC.USA, (2006), pp. $542-545$.

[5] N. Baker, "Zigbee and bluetooth strengths and weaknesses for industrial applications", Computing \& Control Engineering Journal, vol. 16, no. 2, (2005), pp. 20-25. 
[6] X. M. Wang, B. C. Xu and J. J. Chen, "Design and implementation of wireless sensor network node applying to monitoring environment", Measure and Control Technology, vol. 26, no. 11, (2007), pp. 1-3.

[7] L. Li, Y. Zhang and M. H. Wang, "Communication technology for sustainable greenhouse production", Transactions of the Chinese Society for Agricultural Machinery, vol. 38, no. 2, (2007), pp. 195-200.

[8] L. Qu, S. D. Liu and C. B. Hu, "ZigBee technology and application", Bingjing: Beijing University of Aeronautics and Astronautics Press, (2007).

[9] L. Li, M. C. Vurany and I. F. Akyildizy, "Characteristics of Underground Channel for Wireless Underground Sensor Networks", The Sixth Annual Mediterranean Ad Hoc Networking Workshop. Corfu, Greece, vol. 6, (2007), pp. 12-15.

[10] P. Erich, P. D. Stuntebeck and T. Melodia, "Wireless Underground Sensor Networks using Commodity Terrestrial Motes", Wireless Mesh Networks, (2006), pp. 112-114.

[11] R. Aqeel, A. Abu and I. Noman, "A review of wireless sensors and networks' applications in agriculture", Computer Standards \& Interfaces, vol. 1, no. 4, (2011), pp. 259-363.

[12] H. R. Bogena, J. A. Huisman and C. Oberdörster, "Evaluation of a low-cost soil water content sensor for wireless network applications", Journal of Hydrology, vol. 344, (2007), pp. 32-42.

[13] Y. Kima, Y. M. Yangb and W. S. Kanga, "On the Design of Beacon based Wireless Sensor Network for Agricultural Emergency Monitoring Systems”, Computer Standards \& Interfaces, vol. 5, no. 4, (2011), pp. 32-39.

[14] R. J. Bates, "General packet radio service (GPRS) technology and application", Hongbo zhu, translation, Beijing: People's Post and Telecommunication Publishing House, (2004).

[15] ZigBee Alliance, ZigBee specification [EB/OL].http: // www. zigbee. Org., (2008).

[16] G. H. Zhang, L. S. Liu and H. W. Zhang, "The principle and application of 1-wire digital thermometer DS18B20", International Electronic Elements, vol. 14,no. 2, pp. 44-47.

[17] A. S. Chipcon, CC2420 datasheet [EB/OL]. [2004-06-09]. http:\|focus. ti. com/docs/prod/folders/ print/cc2420. html.(2004)

[18] CC2420datasheet, http://focus.ti.com/lit/ds/symlink/cc2420.pdf

[19] J. Q. Xia, J. Yuan and Q. F. Man, "Realization of CAN-Ethernet Communication Module Based on ARM", Measurement \& Control Technology, vol. 23, no. 9, (2004), pp. 30-33.

[20] D. Xie, 'Design of Gateway for Embedded Remote Measurement and Control System Based on ARM", Modern Electronics Technique, vol. 13, (2006), pp. 85-88.

[21] AS Chipcon, "CC2420datasheet [EB/OL]", http:\|focus.ti.com/docs/prod/folders/ print/cc2420.html.

[22] TMEL, ATmega128Ldatasheet [EB/OL]. [2008-06-05]. http: www. atmel. com/ dyn / products/ data sheets. Asp, (2008).

[23] CITECT5.4 manual. CiTechnologies Pty.Limited Commpany, (2002).

\section{Authors}

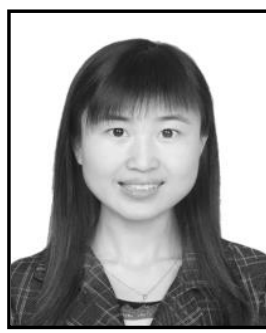

Xiao Q. Yu, she rreceived the B.S. degree from Department of Information Engineering, Lanzhou University of Finance and Economics, Lanzhou, China in 2006. She received her M.S. degree and Ph.D. degree from Department of Mechanical and Electric Engineering and Department of Water Resources and Architectural Engineering, Northwest A \& F University, Shaanxi, China in 2009 and 2013, respectively.

Currently, she is pursuing postdoctoral degree from Department of Water Resources and Architectural Engineering under the supervision of Prof. Wen T. Han. Her current research interests are in Agricultural Water-Soil Engineering and Wireless Sensor Networks. 


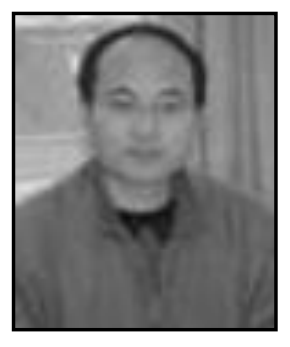

Wen T. Han, he received his B.S. degree from Department of Mechanical and Electric Engineering, Northwest Agriculture University, Shaanxi, China in 1996. M.S. and Ph.D. degree from Department of Mechanical and Electric Engineering, Northwest A \& F University, Shaanxi, China in 1999 and 2004, respectively.

\section{Working Experiences:}

2005-present: researcher, Institute of Soil and Water Conservation of Chinese Academy of Sciences Northwest A \& F University, National Engineering Research Center for Water Saving Irrigation at Yangling.

2004-2005: Assistant Professor, Department of Mechanical and Electric Engineering, Northwest A \& F University.

2001-2004: A lecturer, Department of Mechanical and Electric Engineering, Northwest A \& F University.

\section{Research Interests:}

Information monitoring of crop and environment; Intelligent control for precise irrigation; water distribution Simulation of sprinkler irrigation; Development of nozzle

Currently, he published academic papers more than 30, including SCI and EI articles 16; the national invention patent 6.

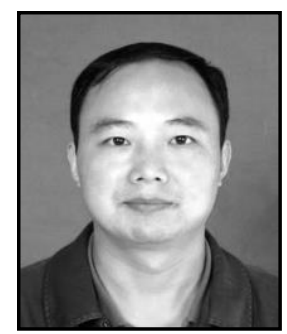

Zeng L. Zhang, he received his B.S. degree from Department of Mechanical and Electric Engineering, Harbin institute of Technology, Harbin, and M.S. degree from Department of Mechanical and Electric Engineering, Northwest A \& F University, Shaanxi, China in 2000 and 2007, respectively.

Currently, he is a teacher in Department of Mechanical and Electric Engineering, Northwest A \& F University, Shaanxi. He is pursuing Ph.D. degree under the supervision of Prof. Pu T. Wu. His current research interests are in Agricultural Water-Soil Engineering and Wireless Sensor Networks. 